Corrigendum

\title{
Corrigendum to "Hierarchical On-line Appearance-Based Tracking for 3D Head Pose, Eyebrows, Lips, Eyelids and Irises" [Image Vision Comput. (2013) 322-340]
}

\author{
Javier Orozco ${ }^{\mathrm{a}, *}$, Ognjen Rudovic $^{\mathrm{a}}$, Jordi Gonzàlez ${ }^{\mathrm{c}}$, Maja Pantic ${ }^{\mathrm{a}, \mathrm{b}}$
}

a Imperial College, Department of Computing, London, UK

${ }^{\mathrm{b}}$ University of Twente, EEMCS, Twente, Netherlands

c Computer Vision Center, Campus UAB, Barcelona, Spain

The authors regret the incorrect insertion of text [totally out of context. make a connection with the previous paragraph] on page 327 (before Eq. (11)).

The authors would like to apologise for any inconvenience caused.

DOI of original article: http://dx.doi.org/10.1016/j.imavis.2013.02.001.

* Corresponding author.

E-mail address: forozcoc@imperial.ac.uk (J. Orozco). 